\title{
AN EXTENSION OF BAIRE'S CATEGORY THEOREM TO RELATOR SPACES
}

\author{
Árpád Száz*
}

\begin{abstract}
As a particular case of a more general result, we show that if $\mathcal{R}$ is a topological, topologically filtered, topologically regular relator on $X$ such that $\mathcal{R}$ is either topologically relatively locally sequentially compact, or uniformly countable and properly sequentially convergence-adherence complete, then $\mathcal{R}$ is a Baire relator.

If $X$ is a nonvoid set, then by a relator $\mathcal{R}$ on $X$ we mean a nonvoid family of binary relations on $X$. The relator $\mathcal{R}$ is called a Baire relator if the fat subsets of the relator space $X(\mathcal{R})$ are not meager. A subset $A$ of $X(\mathcal{R})$ is called fat if $\operatorname{int}_{\mathcal{R}}(A) \neq \emptyset$. While, the set $A$ is called meager if it is a countable union of rare (nowhere dense) sets.
\end{abstract}

\section{A few basic facts on relations and relators}

A subset $F$ of a product set $X \times Y$ is called a relation from $X$ to $Y$. In particular, the relations $\Delta_{X}=\{(x, x) x \in X\}$ and $X^{2}=X \times X$ are called the identity and universal relations on $X$, respectively.

Namely, if in particular $X=Y$, then we may simply say that $F$ is a relation on $X$. Note that if $F$ is a relation from $X$ to $Y$, then $F$ is also a relation on $X \cup Y$. Therefore, it is frequently not a severe restriction to assume that $X=Y$.

If $F$ is a relation on $X$, and moreover $x \in X$ and $A \subset X$, then the sets $F(x)=\{y \in X(x, y) \in F\}$ and $F[A]=\bigcup_{x \in A} F(x)$ are called the images of $x$ and $A$ under $F$, respectively. Whenever $A \in X$ seems unlikely, we may write $F(A)$ in place of $F[A]$.

AMS (MOS) Subject Classification 1991. Primary 54E15, 54E52.

Key words and phrases: Generalized uniformities, Baire's category theorem.

${ }^{*}$ The research of the author has been supported by the grant OTKA T-030082. 
If $F$ is a relation on $X$, then the values $F(x)$, where $x \in X$, uniquely determine $F$ since we have $F=\bigcup_{x \in X}\{x\} \times F(x)$. Therefore, the inverse $F^{-1}$ of $F$ can be defined such that $F^{-1}(x)=\{y \in X: x \in F(y)\}$ for all $x \in X$. Moreover, if $G$ is also a relation on $X$, then the composition $F \circ G$ of $F$ and $G$ can be defined such that $(F \circ G)(x)=F(G(x))$ for all $x \in X$.

A relation $f$ on $X$ is called a function if for each $x \in D_{f}=f^{-1}(X)$ there exists $y \in X$ such that $f(x)=\{y\}$. In this case, by identifying singletons with their elements, we usually write $f(x)=y$. Moreover, sometimes we also write $\left(f_{x}\right)_{x \in D_{f}}=f$ and $\left\{f_{x}\right\}_{x \in D_{f}}=f(X)$, where $f_{x}=f(x)$.

A relation $R$ on $X$ is called reflexive, symmetric, transitive, and directed if $\Delta_{X} \subset R, R=R^{-1}, R \circ R \subset R$, and $X^{2}=R^{-1} \circ R$, respectively. Moreover, a reflexive relation is called a tolerance (preorder) if it is symmetric (transitive). And a directed preorder is called a direction.

If $R$ is a relation on $X$, then we write $R^{n}=R \circ R^{n-1}$ for all $n \in \mathbb{N}$ by agreeing that $R^{0}=\Delta_{X}$. Moreover, we also write $R^{\infty}=\bigcup_{n=0}^{\infty} R^{n}$. Note that thus $R^{\infty}$ is the smallest preorder relation on $X$ such that $R \subset R^{\infty}$.

A nonvoid family $\mathcal{R}$ of relations on a nonvoid set $X$ is called a relator on $X$, and the ordered pair $X(\mathcal{R})=(\mathcal{X}, \mathcal{R})$ is called a relator space. Relator spaces are straightforward generalizations of the various ordered sets and uniform spaces [19]. They deserve to be widely investigated because of the following two facts.

If $\mathcal{D}$ is a nonvoid family of certain distance functions on $X$, then the relator $\mathcal{R}_{\mathcal{D}}$ consisting of all surroundings $B_{r}^{d}=\left\{(x, y) \in X^{2}: d(x, y)<r\right\}$, where $d \in \mathcal{D}$ and $r>0$, is a more convenient mean of defining the basic notions of analysis in the space $X(\mathcal{D})$ than the family of all open subsets of $X(\mathcal{D})$, or even the family $\mathcal{D}$ itself.

Moreover, all reasonable generalizations of the usual topological structures (such as proximities, closures, topologies, filters and convergences, for instance) can be easily derived from relators (according to the results of [23] and [18]), and thus they need not be studied separately.

For instance, if $\mathcal{A}$ is a certain generalized topology or a nonvoid stack (ascending system) in $X$, then $\mathcal{A}$ can be easily derived (according to the forthcoming definitions of the families $\mathcal{T}_{\mathcal{R}}$ and $\mathcal{E}_{\mathcal{R}}$ ) from the Davis-Pervin relator $\mathcal{R}_{\mathcal{A}}$ consisting of all preorders $R_{A}=A^{2} \cup A^{c} \times X$, where $A \in \mathcal{A}$ and $A^{c}=X \backslash A$.

Note that, in contrast to the preorders $R_{A}$, the surroundings $B_{r}^{d}$ are usually tolerances. Therefore, besides preorder relators, tolerance relators are also important particular cases of reflexive relators. Unfortunately, the class of all reflexive relators proved to be inadequate for some important purposes. 


\section{Some important basic tools in relator spaces}

If $\mathcal{R}$ is a relator on $X$, then for any $A, B \subset X$ and $x \in X$ we write:

$$
\begin{gathered}
B \in \operatorname{Int}_{\mathcal{R}}(A) \quad \text { if } \quad R(B) \subset A \text { for some } \quad R \in \mathcal{R}, \\
B \in \mathrm{Cl}_{\mathcal{R}}(A) \text { if } R(B) \cap A \neq \emptyset \text { for all } R \in \mathcal{R} ; \\
x \in \operatorname{int}_{\mathcal{R}}(A) \quad \text { if }\{x\} \in \operatorname{Int}_{\mathcal{R}}(A), \\
x \in \operatorname{cl}_{\mathcal{R}}(A) \text { if }\{x\} \in \operatorname{Cl}_{\mathcal{R}}(A) .
\end{gathered}
$$

Moreover, we also write

$$
\begin{aligned}
& A \in \tau_{\mathcal{R}} \quad \text { if } \quad A \in \operatorname{Int}_{\mathcal{R}}(A), \\
& A \in \mathcal{F}_{\mathcal{R}} \quad \text { if } \quad A^{c} \notin \operatorname{Cl}_{\mathcal{R}}(A) \\
& A \in \mathcal{T}_{\mathcal{R}} \quad \text { if } \quad A \subset \operatorname{int}_{\mathcal{R}}(A), \\
& A \in \mathcal{F}_{\mathcal{R}} \quad \text { if } \quad \operatorname{cl}_{\mathcal{R}}(A) \subset A ; \\
& A \in \mathcal{E}_{\mathcal{R}} \quad \text { if } \quad \operatorname{int}_{\mathcal{R}}(A) \neq \emptyset, \\
& A \in \mathcal{D}_{\mathcal{R}} \quad \text { if } \quad \operatorname{cl}_{\mathcal{R}}(A)=X ;
\end{aligned}
$$

and

$$
\begin{gathered}
A \in \mathcal{N}_{\mathcal{R}} \quad \text { if } \quad \operatorname{cl}_{\mathcal{R}}(A) \notin \mathcal{E}_{\mathcal{R}}, \\
A \in \mathcal{M}_{\mathcal{R}} \quad \text { if } \quad A=\bigcup_{n=1}^{\infty} A_{n}
\end{gathered}
$$

for some sequence $\left(A_{n}\right)_{n=1}^{\infty}$ in $N_{\mathcal{R}}$.

The relations $\operatorname{Int}_{\mathcal{R}}$ and $\operatorname{int}_{\mathcal{R}}$ are called the proximal and topological interiors induced by $\mathcal{R}$, respectively. While, the members of the families $\tau_{\mathcal{R}}$ and $\mathcal{T}_{\mathcal{R}}$ are called the proximally and topologically open subsets of $X(\mathcal{R})$, respectively. And the members of the families $\mathcal{E}_{\mathcal{R}}, \mathcal{N}_{\mathcal{R}}$ and $\mathcal{M}_{\mathcal{R}}$ are called the fat, rare and meager subsets of $X(\mathcal{R})$, respectively.

The fat sets are frequently more important tools than the open sets. For instance, if $\prec$ is a certain order relation on $X$, then $\mathcal{T}_{\prec}$ and $\mathcal{E}_{\prec}$ are just the families of all ascending and residual subsets of the ordered set $X(\prec)$, respectively. Moreover, it may occur that $\mathcal{T}_{\mathcal{R}}=\{\emptyset, X\}$, but $\mathcal{E}_{\mathcal{R}} \neq\{X\}$. Therefore, the fat sets may be useful tools even in a quasi-topologically indiscrete relator space.

A function $x$ of a preordered set $\Gamma$ into $X$ is called a $\Gamma$-net in $X$. The $\Gamma$-net $x$ is said to be eventually (frequently) in a subset $A$ of $X$ if $x^{-1}(A)$ is a fat (dense) subset of $\Gamma$. Therefore, $\Gamma$ could be here an arbitrary relator space. However, preordered nets are usually sufficient.

If $\mathcal{R}$ is a relator on $X$, then for any $\Gamma$-nets $x$ and $y$ in $X$ and $a \in X$ we write:

$$
y \in \operatorname{Lim}_{\mathcal{R}}(x) \quad \text { if the net } \quad(y, x)
$$


is eventually in each $R \in \mathcal{R}$,

$$
y \in \operatorname{Adh}_{\mathcal{R}}(x) \text { if the net }(y, x)
$$

is frequently in each $R \in \mathcal{R}$;

$$
\begin{gathered}
a \in \lim _{\mathcal{R}}(x) \quad \text { if } \quad(a) \in \operatorname{Lim}_{\mathcal{R}}(x), \\
a \in \operatorname{adh}_{\mathcal{R}}(x) \quad \text { if } \quad(a) \in \operatorname{Adh}_{\mathcal{R}}(x) ;
\end{gathered}
$$

where $(a)$ means now the constant net $(a)_{\gamma \in \Gamma}=\Gamma \times\{a\}$. Note that if $\Gamma$ fails to be directed, then $\lim _{\mathcal{R}}(x)$ need not be a subset of $\operatorname{adh}_{\mathcal{R}}(x)$.

\section{Some important unary operations for relators}

If $\mathcal{R}$ is a relator on $X$, then the relators

$$
\begin{gathered}
\mathcal{R}^{*}=\left\{S \subset X^{2}: \exists R \in \mathcal{R}: R \subset S\right\}, \\
\mathcal{R}^{\#}=\left\{S \subset X^{2}: \forall A \subset X: A \in \operatorname{Int}_{\mathcal{R}}(S(A))\right\}, \\
\mathcal{R}^{\wedge}=\left\{S \subset X^{2}: \forall x \in X: x \in \operatorname{int}_{\mathcal{R}}(S(x))\right\}, \\
\mathcal{R}^{\Delta}=\left\{S \subset X^{2}: \forall x \in X: S(x) \in \mathcal{E}_{\mathcal{R}}\right\}
\end{gathered}
$$

are called the uniform, proximal, topological, and paratopological refinements of $\mathcal{R}$, respectively.

On the other hand, the relators

$$
\mathcal{R}^{c}=\left\{R^{c}: R \in \mathcal{R}\right\} \quad \text { and } \quad \mathcal{R}^{-1}=\left\{R^{-1}: R \in \mathcal{R}\right\}
$$

are called the elementwise complement and inverse of $\mathcal{R}$, respectively. Moreover, the relators

$$
\mathcal{R}^{\infty}=\left\{R^{\infty} R \in \mathcal{R}\right\} \quad \text { and } \quad \mathcal{R}^{\partial}=\left\{S \subset X^{2} S^{\infty} \in \mathcal{R}\right\}
$$

are called the direct and inverse preorder modifications of $\mathcal{R}$, respectively. And, for instance, the relators $\mathcal{R}^{\# \partial}=\left(\mathcal{R}^{\#}\right)^{\partial}$ and $\mathcal{R}^{\wedge \infty}=\left(\mathcal{R}^{\wedge}\right)^{\infty}$ are called the super-proximal refinement and quasi-topological modification of $\mathcal{R}$, respectively.

The importance of the above refinement and modification operations is apparent from the following assertions which have been proved in [22], [25], [11] and [14].

Theorem 3.1. If $\mathcal{R}$ is a relator on $X$, then

(1) $\mathcal{R}^{*}$ is the largest relator on $X$ such that $\operatorname{Lim}_{\mathcal{R}}=\operatorname{Lim}_{\mathcal{R}^{*}}$, or equivalently $\operatorname{Adh}_{\mathcal{R}}=\operatorname{Adh}_{\mathcal{R}^{*}}$;

(2) $\mathcal{R}^{\#}$ is the largest relator on $X$ such that $\operatorname{Int}_{\mathcal{R}}=\operatorname{Int}_{\mathcal{R}^{\#}}$, or equivalently $\mathrm{Cl}_{\mathcal{R}}=\mathrm{Cl}_{\mathcal{R}^{\#}}$; 
(3) $\mathcal{R}^{\wedge}$ is the largest relator on $X$ such that $\lim _{\mathcal{R}}=\lim _{\mathcal{R}^{\wedge}}$, or equivalently $\operatorname{adh}_{\mathcal{R}}=\operatorname{adh}_{\mathcal{R}^{\wedge}}$;

(4) $\mathcal{R}^{\wedge}$ is the largest relator on $X$ such that $\operatorname{int}_{\mathcal{R}}=\operatorname{int}_{\mathcal{R}^{\wedge}}$, or equivalently $\mathrm{cl}_{\mathcal{R}}=\operatorname{cl}_{\mathcal{R}^{\wedge}}$;

(5) $\mathcal{R}^{\Delta}$ is the largest relator on $X$ such that $\mathcal{E}_{\mathcal{R}}=\mathcal{E}_{\mathcal{R}^{\Delta}}$, or equivalently $\mathcal{D}_{\mathcal{R}}=\mathcal{D}_{\mathcal{R}^{\Delta}}$

(6) $\mathcal{R}^{\# \partial}$ is the largest relator on $X$ such that $\tau_{\mathcal{R}}=\tau_{\mathcal{R} \# \partial}$, or equivalently $\tau_{\mathcal{R}}=\tau_{\mathcal{R} \# \partial} ;$

(7) $\mathcal{R}^{\wedge \infty}$ is the largest preorder relator on $X$ such that $\mathcal{T}_{\mathcal{R}}=\mathcal{T}_{\mathcal{R}^{\wedge \infty}}$, or equivalently $\mathcal{F}_{\mathcal{R}}=\mathcal{F}_{\mathcal{R}^{\wedge \infty}}$.

Remark 3.2. If $\mathcal{R}$ is a relator on $X$, then in general there does not exist a largest relator $\mathcal{R}^{\square}$ such that $\mathcal{T}_{\mathcal{R}}=\mathcal{T}_{\mathcal{R}}$.

In this respect, it is also worth mentioning that the operation \# $\#$ is not stable in the sense that in general $\left\{X^{2}\right\}^{\# \partial} \neq\left\{X^{2}\right\}$.

Moreover, the operations $\wedge$ and $\Delta$ are not inversion compatible. Therefore, sometimes we shall also need the relators

$$
\mathcal{R}^{\vee}=\left(\mathcal{R}^{\wedge}\right)^{-1} \quad \text { and } \quad \mathcal{R}^{\nabla}=\left(\mathcal{R}^{\triangle}\right)^{-1} .
$$

Remark 3.3. Because of Theorem 3.1, two relators $\mathcal{R}$ and $\mathcal{S}$ on $X$ may, for instance, be called topologically equivalent if $\mathcal{R}^{\wedge}=\mathcal{S}^{\wedge}$. And the relator $\mathcal{R}$ may be called topologically fine if $\mathcal{R}^{\wedge}=\mathcal{R}$.

Moreover, the relator $\mathcal{R}$ may be called topologically simple (topologically countable) if it is topologically equivalent to a singleton (countable) relator. Therefore, a singleton (countable) relator will be called properly simple (properly countable).

Remark 3.4. Finally, we note that besides the above basic unary operations it is also convenient to consider some binary operations for relators.

For instance, for any relators $\mathcal{R}$ and $\mathcal{S}$ on $X$, we may naturally write

$$
\mathcal{R} \circ \mathcal{S}=\{R \circ S R \in \mathcal{R}, S \in \mathcal{S}\} \quad \text { and } \quad \mathcal{R} \wedge \mathcal{S}=\{R \cap S R \in \mathcal{R}, S \in \mathcal{S}\} .
$$

Note that thus we have $\mathcal{R} \cap \mathcal{S} \subset \mathcal{R} \wedge \mathcal{S}$ and $\mathcal{R} \cup \mathcal{S} \subset(\mathcal{R} \wedge \mathcal{S})^{*}$, for instance.

\section{Reflexive and topological relators}

Definicija 4.1. If $\mathcal{R}$ is a relator on $X$, then we say that:

(1) $\mathcal{R}$ is reflexive if $x \in R(x)$ for all $x \in X$ and $R \in \mathcal{R}$;

(2) $\mathcal{R}$ is quasi-topological if $x \in \operatorname{int}_{\mathcal{R}}\left(\operatorname{int}_{\mathcal{R}}(R(x))\right)$ for all $x \in X$ and $R \in \mathcal{R}$

(3) $\mathcal{R}$ is topological if for all $x \in X$ and $R \in \mathcal{R}$ there exists $V \in \mathcal{T}_{\mathcal{R}}$ such that $x \in V \subset R(x)$. 
Remark 4.2. Note that the inclusion $x \in \operatorname{int}_{\mathcal{R}}(R(x))$ trivially holds for all $x \in X$ and $R \in \mathcal{R}$.

Moreover, the relator $\mathcal{R}$ may be called proximal if for any $A \subset X$ and $R \in \mathcal{R}$ there exists $V \in \tau_{\mathcal{R}}$ such that $A \subset V \subset R(A)$.

The appropriateness of Definition 3.1 is quite obvious from the following three basic theorems which have been proved in [20], [22] and [28].

Theorem 4.3. If $\mathcal{R}$ is a relator on $X$, then the following assertions are equivalent:

(1) $\mathcal{R}$ is reflexive;

(2) $\cap \mathcal{R}$ is reflexive;

(3) $\operatorname{int}_{\mathcal{R}}(A) \subset A$ for all $A \subset X$;

(4) $A \subset \operatorname{cl}_{\mathcal{R}}(A)$ for all $A \subset X$.

Remark 4.4. The reflexivity of the relator $\mathcal{R}$ can also be nicely characterized in terms of the relations $\operatorname{Int}_{\mathcal{R}}, \mathrm{Cl}_{\mathcal{R}}, \operatorname{Lim}_{\mathcal{R}}$ and $\mathrm{Adh}_{\mathcal{R}}$.

For instance, we can easily see that $\mathcal{R}$ is reflexive if and only if $\operatorname{Int}_{\mathcal{R}}(A) \subset$ $\mathcal{P}(A)$ for all $A \subset X$. Hence, we can immediately infer that $\operatorname{Int}_{\mathcal{R}}$ is transitive whenever $\mathcal{R}$ is reflexive.

Theorem 4.5. If $\mathcal{R}$ is a relator on $X$, then the following assertions are equivalent:

(1) $\mathcal{R}$ is quasi-topological;

(2) $\operatorname{int}_{\mathcal{R}}(R(x)) \in \mathcal{T}_{\mathcal{R}}$ for all $x \in X$ and $R \in \mathcal{R}$;

(3) $\operatorname{int}_{\mathcal{R}}(A) \in \mathcal{T}_{\mathcal{R}}$ for all $A \subset X$;

(4) $\operatorname{cl}_{\mathcal{R}}(A) \in \mathcal{F}_{\mathcal{R}}$ for all $A \subset X$.

Theorem 4.6. If $\mathcal{R}$ is a relator on $X$, then the following assertions are equivalent:

(1) $\mathcal{R}$ is topological;

(2) $\mathcal{R}$ is reflexive and quasi-topological;

(3) $\operatorname{int}_{\mathcal{R}}(A)=\bigcup\left\{V \in \mathcal{T}_{\mathcal{R}}: V \subset A\right\}$ for all $A \subset X$;

(4) $\operatorname{cl}_{\mathcal{R}}(A)=\bigcap\left\{W \in \mathcal{F}_{\mathcal{R}}: A \subset W\right\}$ for all $A \subset X$.

In view of the latter two theorems, we may also naturally introduce the following

Definicija 4.7. A relator $\mathcal{R}$ on $X$ is called weakly (strongly) quasitopological if $\operatorname{cl}_{\mathcal{R}}(\{x\}) \in \mathcal{F}_{\mathcal{R}}$ for all $x \in X\left(R(x) \in \mathcal{T}_{\mathcal{R}}\right.$ for all $x \in X$ and $R \in \mathcal{R})$.

Moreover, the relator $\mathcal{R}$ is called weakly (strongly) topological if it is reflexive and weakly (strongly) quasi-topological. 
The importance of strongly topological relators lies mainly in the following theorem which has also been proved in [20], [22] and [28].

Theorem 4.8. If $\mathcal{R}$ is a relator on $X$ and $R^{\circ}$ is a relation on $X$ for each $R \in \mathcal{R}$ such that

$$
R^{\circ}(x)=\operatorname{int}_{\mathcal{R}}(R(x))
$$

for all $x \in X$, then $\mathcal{R}^{\circ}=\left\{R^{\circ}: R \in \mathcal{R}\right\}$ is a strongly topological relator on $X$ such that:

(1) $\mathcal{R}$ is quasi-topological $\Longleftrightarrow \mathcal{R}^{\circ} \subset \mathcal{R}^{\wedge}$;

(2) $\mathcal{R}$ is reflexive $\Longleftrightarrow \mathcal{R} \subset\left(\mathcal{R}^{\circ}\right)^{*} \Longleftrightarrow \mathcal{R} \subset\left(\mathcal{R}^{\circ}\right)^{\wedge}$.

Now, as an immediate consequence of the above theorem and the topological invariance of topologicalness, we can also state

Corollary 4.9. If $\mathcal{R}$ is a relator on $X$, then the following assertions are equivalent:

(1) $\mathcal{R}$ is topological;

(2) $\mathcal{R}^{\wedge}=\left(\mathcal{R}^{\circ}\right)^{\wedge}$.

In this respect, it is also worth mentioning that we also have the following

Theorem 4.10. If $\mathcal{R}$ is a relator on $X$, then the following assertions are equivalent:

(1) $\mathcal{R}$ is topological;

(2) $\mathcal{R}^{\wedge}=\left(\mathcal{R}_{\mathcal{T}_{\mathcal{R}}}\right)^{\wedge}$

(3) $\mathcal{R}^{\wedge}=\left(\mathcal{R}^{\wedge \infty}\right)^{\wedge}$;

(4) $\mathcal{R}$ is topologically equivalent to a preorder relator.

Remark 4.11. Note that if $\mathcal{T}$ is a topology on $X$, then the preorder relator $\mathcal{R}_{\mathcal{T}}$ is not, in general, weakly symmetric. If $\mathcal{D}$ is a nonvoid family of pseudo-metrics on $X$, then the tolerance relator $\mathcal{R}_{\mathcal{D}}$ is not, in general, strongly transitive.

\section{Symmetric, transitive and regular relators}

Definicija 5.1. If $\mathcal{R}$ is a relator on $X$, then we say that:

(1) $\mathcal{R}$ is properly symmetric if $\mathcal{R}^{-1} \subset \mathcal{R}$;

(2) $\mathcal{R}$ is topologically symmetric if $\mathcal{R}^{\vee} \subset \mathcal{R}^{\wedge}$;

(3) $\mathcal{R}$ is topologically semisymmetric if $\mathcal{R}^{-1} \subset \mathcal{R}^{\wedge}$.

Remark 5.2. The relator $\mathcal{R}$ may be called weakly (strongly) symmetric if $\bigcap \mathcal{R}$ ( each member of $\mathcal{R}$ ) is symmetric. 
The following theorem of [10] shows that topological symmetry is a rather restrictive property.

Theorem 5.3. If $\mathcal{R}$ is a relator on $X$, then the following assertions are equivalent:

(1) $\mathcal{R}$ is topologically symmetric;

(2) $\mathcal{R}$ is topologically simple and weakly symmetric.

Definicija 5.4. If $\mathcal{R}$ is a relator on $X$, then we say that:

(1) $\mathcal{R}$ is uniformly transitive if $\mathcal{R} \subset(\mathcal{R} \circ \mathcal{R})^{*}$;

(2) $\mathcal{R}$ is topologically transitive if $\mathcal{R} \subset\left(\mathcal{R}^{\wedge} \circ \mathcal{R}\right)^{\wedge}$;

(3) $\mathcal{R}$ is strongly topologically transitive if $\mathcal{R} \subset(\mathcal{R} \circ \mathcal{R})^{\wedge}$;

The following theorem of [22] shows that, in contrast to topological symmetry, topological transitivity is not a very restrictive property.

Theorem 5.5. If $\mathcal{R}$ is a reflexive relator on $X$, then the following assertions are equivalent:

(1) $\mathcal{R}$ is quasi-topological;

(2) $\mathcal{R}$ is topologically transitive.

As a combination of topological symmetry and topological transitivity, we may also naturally introduce the following

Definicija 5.6. A relator $\mathcal{R}$ on $X$ is called topologically regular if

$$
\mathcal{R} \subset\left(\mathcal{R}^{\vee} \circ \mathcal{R}\right)^{\wedge}
$$

where $\mathcal{R}^{\vee}=\left(\mathcal{R}^{\wedge}\right)^{-1}$.

The appropriateness of this notion is apparent from following theorem of $[17]$.

Theorem 5.7. If $\mathcal{R}$ is a relator on $X$, then the following assertions are equivalent:

(1) $\mathcal{R}$ is topologically regular;

(2) for any $x \in X$ and $A \subset X$ with $x \notin \operatorname{cl}_{\mathcal{R}}(A)$ there exists $U \in \mathcal{R}$ and $V \in \mathcal{R}^{\wedge}$ such that $U(x) \cap V(A)=\emptyset$.

Moreover, improving some theorems of [20], [28] and [30], we can also easily prove the following

Theorem 5.8. If $\mathcal{R}$ is a relator on $X$ and $R^{-}$is a relation on $X$ for each $R \in \mathcal{R}$ such that

$$
R^{-}(x)=\operatorname{cl}_{\mathcal{R}}(R(x))
$$

for all $x \in X$, then $\mathcal{R}^{-}=\left\{R^{-}: R \in \mathcal{R}\right\}$ is a relator on $X$ such that:

(1) $\mathcal{R}$ is reflexive $\Longrightarrow \mathcal{R}^{-} \subset \mathcal{R}^{*}$;

(2) $R$ is topologically regular $\Longleftrightarrow \mathcal{R} \subset\left(\mathcal{R}^{-}\right)^{\wedge}$. 
Hint. To prove the "if part" of (2), note that if $\mathcal{R} \subset\left(\mathcal{R}^{-}\right)^{\wedge}$, then for any $x \in X$ and $R \in \mathcal{R}$ there exists $U \in \mathcal{R}$ such that $\operatorname{cl}_{\mathcal{R}}(U(x)) \subset R(x)$, and thus $R(x)^{c} \subset \operatorname{cl}_{\mathcal{R}}(U(x))^{c}$. Therefore, for each $y \in R(x)^{c}$ there exists $V_{y} \in \mathcal{R}$ such that $V_{y}(y) \cap U(x)=\emptyset$. Define a relation $V$ on $X$ such that $V(y)=X$ for all $y \in R(x)$ and $V(y)=V_{y}(y)$ for $y \in R(x)^{c}$. Then, it is clear that $V \in \mathcal{R}^{\wedge}$ such that $V(y) \cap U(x)=\emptyset$, and thus $y \notin V^{-1}(U(x))$ for all $y \in R(x)^{c}$. Hence, it follows that $V^{-1}(U(x)) \subset R(x)$. Therefore, $\mathcal{R} \subset\left(\mathcal{R}^{\vee} \circ \mathcal{R}\right)^{\wedge}$, and thus $\mathcal{R}$ is topologically regular.

Now, as an immediate consequence of the above theorem and the topological invariance of reflexivity and topological regularity, we can also state

Corollary 5.9. If $\mathcal{R}$ is a reflexive, topologically regular relator on $X$, and then $\mathcal{R}^{-}$is a reflexive, topologically regular relator on $X$ such that $\mathcal{R}^{\wedge}=$ $\left(\mathcal{R}^{-}\right)^{\wedge}$.

Remark 5.10. Concerning the above definitions, it is also worth mentioning that if $\mathcal{R}$ is topologically symmetric and topologically transitive, or $\mathcal{R}$ is topologically semisymmetric and strongly topologically transitive, then $\mathcal{R}$ is already topologically regular.

On the other hand, if $\mathcal{R}$ is topologically symmetric and topologically regular, then $\mathcal{R}$ is topologically transitive. Moreover, if $\mathcal{R}$ is reflexive and topologically regular, then $\mathcal{R}$ weakly symmetric. (This depends on the fact that $\mathcal{R}^{\vee}$ is always topologically simple.)

Definicija 5.11. If $\mathcal{R}$ is a relator on $X$, then we say that:

(1) $\mathcal{R}$ is properly filtered if $\mathcal{R} \wedge \mathcal{R} \subset \mathcal{R}$;

(2) $\mathcal{R}$ is uniformly filtered if $\mathcal{R} \wedge \mathcal{R} \subset \mathcal{R}^{*}$;

(3) $\mathcal{R}$ is topologically filtered if $\mathcal{R} \wedge \mathcal{R} \subset \mathcal{R}^{\wedge}$.

Remark 5.12. Moreover, the relator $\mathcal{R}$ may, for instance, be called totally filtered if for any $R, S \in \mathcal{R}$ we have either $R \subset S$ or $S \subset R$.

The importance of topological filteredness is apparent from the following theorem of [20] and [22].

Theorem 5.13. If $\mathcal{R}$ is a relator on $X$, then the following assertions are equivalent:

(1) $\mathcal{R}$ is topologically filtered;

(2) $\operatorname{cl}_{\mathcal{R}}(A \cup B)=\operatorname{cl}_{\mathcal{R}}(A) \cup \operatorname{cl}_{\mathcal{R}}(B)$ for all $A, B \subset X$;

(3) $\operatorname{int}_{\mathcal{R}}(A \cap B)=\operatorname{int}_{\mathcal{R}}(A) \cap \operatorname{int}_{\mathcal{R}}(B)$ for all $A, B \subset X$.

Corollary 5.14. If $\mathcal{R}$ is a topologically filtered relator on $X$, then the families $\mathcal{T}_{\mathcal{R}}$ and $\mathcal{F}_{\mathcal{R}}$ are closed under finite intersections and unions, respectively. 


\section{Cauchy nets and completeness in relator spaces}

Definicija 6.1. A net $x$ in a relator space $X(\mathcal{R})$ is called convergent (adherent) if $\lim _{\mathcal{R}}(x) \neq \emptyset\left(\operatorname{adh}_{\mathcal{R}}(x) \neq \emptyset\right)$.

Moreover, a net $x$ in a relator space $X(\mathcal{R})$ is called properly convergence (adherence) Cauchy if it is convergent (adherent) in each of the simple relator spaces $X(R)$ where $R \in \mathcal{R}$.

Remark 6.2. Since

$$
\lim _{\mathcal{R}}(x)=\bigcap_{R \in \mathcal{R}} \lim _{R}(x)\left(\operatorname{adh}_{\mathcal{R}}(x)=\bigcap_{R \in \mathcal{R}} \operatorname{adh}_{R}(x)\right),
$$

it is clear that a convergent (adherent) net is in particular properly convergence (adherence) Cauchy.

Moreover, by calling a net $x$ in a relator space $X(\mathcal{R})$ topologically convergence (adherence) Cauchy if it is properly convergence (adherence) Cauchy in the space $X\left(\mathcal{R}^{\wedge}\right)$, we can easily establish the following extension of [26, Theorem 2.1].

Theorem 6.3. If $x$ is a $\Gamma$-net in a relator space $X(\mathcal{R})$, then the following assertions are equivalent:

(1) $x$ is convergent (adherent);

(2) $x$ is topologically convergence (adherence) Cauchy.

Remark 6.4. Therefore, the notions of a convergent (adherent) net and a convergence (adherence) Cauchy net are, in a certain sense, equivalent.

Moreover, it is also worth mentioning that the notion of a convergence (adherence) Cauchy net is also closely related to the notion of an infinitesimal family.

Definicija 6.5. A family $\mathcal{A}$ of subsets of a relator space $X(\mathcal{R})$ is called infinitesimal if for each $R \in \mathcal{R}$ there exist $x \in X$ and $A \in \mathcal{A}$ such that $A \subset R(x)$.

Remark 6.6. Now, an indexed family $\left(A_{i}\right)_{i \in I}$ of subsets of $X(\mathcal{R})$ may be called infinitesimal if the unindexed family $\left\{A_{i}\right\}_{i \in I}$ is infinitesimal.

Moreover, by using the notation $x(\mathcal{A})=\{x(A)\}_{A \in \mathcal{A}}$ for any $\Gamma$-net $x$ in $X$ and $A \subset \mathcal{P}(\Gamma)$, we can easily establish the following

Theorem 6.7. If $x$ is a $\Gamma$-net in a relator space $X(\mathcal{R})$, then the following assertions are equivalent:

(1) $x$ is properly convergence (adherence) Cauchy;

(2) the family $x\left(\mathcal{E}_{\Gamma}\right)\left(x\left(\mathcal{D}_{\Gamma}\right)\right)$ is infinitesimal. 
Definicija 6.8. An ordered pair $(\mathcal{R}, \mathcal{S})$ of relators on $X$ is called properly sequentially convergence-adherence complete if every properly convergence Cauchy sequence in $X(\mathcal{S})$ is adherent in $X(\mathcal{R})$.

Remark 6.9. Now, by identifying singletons with their elements, a relator $\mathcal{R}$ on $X$ may be naturally called properly sequentially convergenceadherence complete if the pair $(\mathcal{R}, \mathcal{R})$ has the corresponding property.

By Theorems 6.3 and 3.1, it is clear that a pair $(\mathcal{R}, \mathcal{S})$ of relators on $X$ with $\mathcal{R} \subset \mathcal{S}^{\wedge}$ is already topologically sequentially convergence-adherence complete in the sense that the pair $\left(\mathcal{R}, \mathcal{S}^{\wedge}\right)$ is properly sequentially convergenceadherence complete.

Moreover, by calling a family $\mathcal{A}$ of subsets of $X$ centered [4, p. 57] if $\bigcap \mathcal{B} \neq \emptyset$ for any finite subfamily $\mathcal{B}$ of $\mathcal{A}$, we can easily prove the following analogue of [26, Theorem 4.2].

Theorem 6.10. If $(\mathcal{R}, \mathcal{S})$ is a pair of relators on $X$, then the following assertions are equivalent:

(1) $(\mathcal{R}, \mathcal{S})$ is properly sequentially convergence-adherence complete;

(2) $\bigcap_{n=1}^{\infty} \operatorname{cl}_{\mathcal{R}}\left(A_{n}\right) \neq \emptyset$ for any decreasing, infinitesimal sequence $\left(A_{n}\right)_{n=1}^{\infty}$ of nonvoid subsets of $X(\mathcal{S})$;

(3) $\bigcap_{A \in \mathcal{A}} \mathrm{cl}_{\mathcal{R}}(A) \neq \emptyset$ for any countable, centered, infinitesimal family $\mathcal{A}$ of subsets of $X(\mathcal{S})$.

Hint To prove the implication $(1) \Longrightarrow(2)$, note that if $\left(A_{n}\right)_{n=1}^{\infty}$ is as in the assertion (2), then by choosing $x_{n} \in A_{n}$ for each $n \in \mathbb{N}$ we can get a properly convergence Cauchy sequence $\left(x_{n}\right)_{n=1}^{\infty}$ in $X(\mathcal{S})$ such that

$$
\operatorname{adh}_{\mathcal{R}}\left(\left(x_{n}\right)_{n=1}^{\infty}\right)=\bigcap_{n=1}^{\infty} \operatorname{cl}_{\mathcal{R}}\left(\left\{x_{k}\right\}_{k=n}^{\infty}\right) \subset \bigcap_{n=1}^{\infty} \operatorname{cl}_{\mathcal{R}}\left(A_{n}\right) .
$$

Therefore, if the assertion (1) holds, then we have not only $\operatorname{adh}_{\mathcal{R}}\left(\left(x_{n}\right)_{n=1}^{\infty}\right) \neq$ $\emptyset$, but also $\bigcap_{n=1}^{\infty} \operatorname{cl}_{\mathcal{R}}\left(A_{n}\right) \neq \emptyset$.

\section{Baire complete relators}

Definicija 7.1. A relator $\mathcal{R}$ on $X$ is called Baire complete if there exists a properly countable relator $\mathcal{S}$ on $X$ with $\mathcal{S} \subset \mathcal{R}^{\wedge}$ such that the pair $(\mathcal{R}, \mathcal{S})$ is properly sequentially convergence-adherence complete.

Remark 7.2. Baire completeness seems to be the natural counterpart of the weak hypocompactness of Császár [4, p. 388] which is a generalization of Čech completeness studied by several authors. (See [6, p. 256].) 
Theorem 7.3. If $\mathcal{R}$ is a uniformly countable and properly sequentially convergence-adherence complete relator on $X$, then $\mathcal{R}$ is Baire complete.

Proof. Since $\mathcal{R}$ is uniformly countable, there exists a properly countable relator $\mathcal{S}$ on $X$ such that $\mathcal{R}^{*}=\mathcal{S}^{*}$. This implies that $\mathcal{R} \subset \mathcal{S}^{*}$. Therefore, for each $R \in \mathcal{R}$ there exists $S \in \mathcal{S}$ such that $S \subset R$. Hence, it is clear that if $\left(x_{n}\right)_{n=1}^{\infty}$ is a properly convergence Cauchy sequence in $X(\mathcal{S})$, then $\left(x_{n}\right)_{n=1}^{\infty}$ is also properly convergence Cauchy in $X(\mathcal{R})$. Hence, by the assumed completeness property of $\mathcal{R}$, it follows that $\left(x_{n}\right)_{n=1}^{\infty}$ is adherent in $X(\mathcal{R})$. Therefore, the pair $(\mathcal{R}, \mathcal{S})$ is properly sequentially convergence-adherence complete. Hence, since $\mathcal{R}^{*}=\mathcal{S}^{*}$ also implies $\mathcal{S} \subset \mathcal{R}^{\wedge}$, it is clear that $\mathcal{R}$ is Baire complete.

Now, as a simple application of the above theorem, we can also state

Corollary 7.4. If $d$ is complete pseudo-metric on $X$ in the usual sense, then $\mathcal{R}_{d}$ is a Baire complete relator on $X$.

Proof. Note that $\mathcal{R}_{d}=\left\{B_{r}^{d}: r>0\right\}$ is now a properly sequentially convergence-adherence complete relator on $X$ such that under the notation $\mathcal{S}=\left\{B_{1 / n}^{d}: n \in \mathbb{N}\right\}$ we have $\mathcal{R}^{*}=\mathcal{S}^{*}$. Therefore, Theorem 7.3 can be applied.

Because of [29, Theorem 4.1], we may also naturally introduce the following

Definicija 7.5. A subset $Y$ of a relator space $X(\mathcal{R})$ is called properly relatively sequentially compact if every sequence $\left(x_{n}\right)_{n=1}^{\infty}$ in $Y$ is properly adherence Cauchy in $X(\mathcal{R})$.

Now, by calling a subset $Y$ of a relator space $X(\mathcal{R})$ topologically relatively sequentially compact if it is properly relatively sequentially compact in the relator space $X\left(\mathcal{R}^{\wedge}\right)$, we can easily establish the following consequence of Theorem 6.3.

Theorem 7.6. If $Y$ is a subset of a relator space $X(\mathcal{R})$, then the following assertions equivalent:

(1) $Y$ is topologically relatively sequentially compact;

(2) each sequence $\left(x_{n}\right)_{n=1}^{\infty}$ in $Y$ is adherent in $X(\mathcal{R})$.

Moreover, analogously to [29, Theorems 5.1 and 5.2], we can also easily establish the following

Theorem 7.7. If $Y$ is a subset of a relator space $X(\mathcal{R})$, then the following assertions are equivalent:

(1) $Y$ is topologically relatively sequentially compact;

(2) $\bigcap_{n=1}^{\infty} \operatorname{cl}_{\mathcal{R}}\left(A_{n}\right) \neq \emptyset$ for any decreasing sequence $\left(A_{n}\right)_{n=1}^{\infty}$ of nonvoid subsets of $Y$;

(3) $\bigcap_{A \in \mathcal{A}} \operatorname{cl}_{\mathcal{R}}(A) \neq \emptyset$ for any countable centered family $\mathcal{A}$ of subsets of $Y$. 
Definicija 7.8. A relator $\mathcal{R}$ on $X$ is called properly relatively locally sequentially compact if for each $x \in X$ there exists $R \in \mathcal{R}$ such that $R(x)$ is properly relatively sequentially compact in $X(\mathcal{R})$.

Now, by calling a relator $\mathcal{R}$ on $X$ topologically relatively locally sequentially compact if the relator $\mathcal{R}^{\wedge}$ is properly relatively locally sequentially compact, we can easily establish the following

Theorem 7.9. If $\mathcal{R}$ is a relator on $X$, then the following assertions are equivalent:

(1) $\mathcal{R}$ is topologically relatively locally sequentially compact;

(2) for each $x \in X$ there exists $R \in \mathcal{R}$ such that $R(x)$ is topologically relatively sequentially compact in $X(\mathcal{R})$.

However, the importance of topologically relatively sequentially compact relators lies mainly in the following

Theorem 7.10. If $\mathcal{R}$ is a topologically relatively locally sequentially compact relator on $X$, then $\mathcal{R}$ is Baire complete.

Proof. In this case, by Theorem 7.9, for each $x \in X$ there exists $S_{x} \in$ $\mathcal{R}$ such that $S_{x}(x)$ is topologically relatively sequentially compact in $X(\mathcal{R})$. Define a relation $S$ on $X$ such that $S(x)=S_{x}(x)$ for all $x \in X$. Then, it is clear that $\mathcal{S}=\{S\}$ is a countable relator on $X$ such that $\mathcal{S} \subset \mathcal{R}^{\wedge}$. Moreover, we can also easily see that the pair $(\mathcal{R}, \mathcal{S})$ is properly sequentially convergence-adherence complete. Therefore, $\mathcal{R}$ is Baire complete.

Namely, if $\left(x_{n}\right)_{n=1}^{\infty}$ is a properly convergence Cauchy sequence in $X(\mathcal{S})$, then by the corresponding definitions there exist $x \in X$ and $n_{0} \in \mathbb{N}$ such that the sequence $\left(x_{n_{0}+n}\right)_{n=1}^{\infty}$ is in $S(x)=S_{x}(x)$. Hence, by Theorem 7.6, it follows that $\operatorname{adh}_{\mathcal{R}}\left(\left(x_{n}\right)_{n=1}^{\infty}\right)=\operatorname{adh}_{\mathcal{R}}\left(\left(x_{n_{0}+n}\right)_{n=1}^{\infty}\right) \neq \emptyset$. Therefore, the sequence $\left(x_{n}\right)_{n=1}^{\infty}$ is adherent in $X(\mathcal{R})$.

\section{An extension of Baire's category theorem}

Definicija 8.1. A relator $\mathcal{R}$ on $X$ is called a Baire relator if

$$
\mathcal{E}_{\mathcal{R}} \cap \mathcal{M}_{\mathcal{R}}=\emptyset .
$$

Remark 8.2. Note that thus a relator space $X(\mathcal{R})$ is a Baire relator space if and only if the fat subsets of $X(\mathcal{R})$ are not meager.

Moreover, as a simple, but important characterization of Baire relator, we can easily establish the following theorem of [33]. 
Theorem 8.3. If $\mathcal{R}$ is a relator on $X$, then the following assertions are equivalent:

(1) $\mathcal{R}$ is a Baire relator;

(2) if $\bigcup_{i=1}^{\infty} A_{n} \in \mathcal{E}_{\mathcal{R}}$, then $\operatorname{cl}_{\mathcal{R}}\left(A_{n}\right) \in \mathcal{E}_{\mathcal{R}}$ for some $n \in \mathbb{N}$;

(3) if $\operatorname{int}_{\mathcal{R}}\left(A_{n}\right) \in \mathcal{D}_{\mathcal{R}}$ for all $n \in \mathbb{N}$, then $\bigcap_{n=1}^{\infty} A_{n} \in \mathcal{D}_{\mathcal{R}}$.

Now, having all the necessary preparations, we are ready to prove the following generalization of Baire's category theorem [2, p. 193].

Theorem 8.4. If $\mathcal{R}$ is a topological, topologically filtered, topologically regular and Baire complete relator on $X$, then $\mathcal{R}$ is a Baire relator.

Proof. Note that, by Theorem 4.8, we may assume without loss of generality that the relator $\mathcal{R}$ is strongly topological. Namely, all the properties occurring in the theorem can easily be seen to be topologically invariant. Moreover, by Theorem 9.3 , it is enough to prove only that if $\left(A_{n}\right)_{n=1}^{\infty}$ is a sequence of subsets of $X$ such that $\operatorname{int}_{\mathcal{R}}\left(A_{n}\right) \in \mathcal{D}_{\mathcal{R}}$ for all $n \in \mathbb{N}$, then $A=\bigcap_{n=1}^{\infty} A_{n} \in \mathcal{D}_{\mathcal{R}}$. That is, for any $x_{0} \in X$ and $U_{0} \in \mathcal{R}$ we have $A \cap$ $U_{0}\left(x_{0}\right) \neq \emptyset$. For this, note that by the Baire completeness of $\mathcal{R}$ there exists a countable relator $\mathcal{S}=\left\{S_{n}\right\}_{n=1}^{\infty}$ on $X$ with $\mathcal{S} \subset \mathcal{R}^{\wedge}$ such that the pair $(\mathcal{R}, \mathcal{S})$ is properly sequentially convergence-adherence complete. Moreover, since $\operatorname{int}_{\mathcal{R}}\left(A_{1}\right) \in \mathcal{D}_{\mathcal{R}}$, there exists $x_{1} \in X$ such that

$$
x_{1} \in \operatorname{int}_{\mathcal{R}}\left(A_{1}\right) \quad \text { and } \quad x_{1} \in U_{0}\left(x_{0}\right) .
$$

Thus, by the definition of the relation $\operatorname{int}_{\mathcal{R}}$ and the strong quasi-topologicalness of $\mathcal{R}$, there exist $V_{1} \in \mathcal{R}$ and $V_{2} \in \mathcal{R}$ such that

$$
V_{1}\left(x_{1}\right) \subset A_{1} \quad \text { and } \quad V_{2}\left(x_{1}\right) \subset U_{0}\left(x_{0}\right) .
$$

Moreover, since $S_{1} \in \mathcal{R}^{\wedge}$ and $\mathcal{R}$ is topologically filtered, there exist $V_{3} \in \mathcal{R}$ and $V_{4} \in \mathcal{R}$ such that

$$
V_{3}\left(x_{1}\right) \subset S_{1}\left(x_{1}\right) \quad \text { and } \quad V_{4}\left(x_{1}\right) \subset V_{1}\left(x_{1}\right) \cap V_{2}\left(x_{1}\right) \cap V_{3}\left(x_{1}\right) .
$$

On the other hand, since $\mathcal{R}$ is topologically regular, by Theorem 5.8 there exists $U_{1} \in \mathcal{R}$ such that

$$
\operatorname{cl}_{\mathcal{R}}\left(U_{1}\left(x_{1}\right)\right) \subset V_{4}\left(x_{1}\right) .
$$

Now, in conclusion of the above argument, we can state that there exist $x_{1} \in X$ and $U_{1} \in \mathcal{R}$ such that

$$
\operatorname{cl}_{\mathcal{R}}\left(U_{1}\left(x_{1}\right)\right) \subset A_{1} \cap U_{0}\left(x_{0}\right) \cap S_{1}\left(x_{1}\right) .
$$

Moreover, by repeating the above argument with $x_{1}, U_{1}, A_{2}$ and $S_{2}$ in place of $x_{0}, U_{0}, A_{1}$ and $S_{1}$, we can see that there exist $x_{2} \in X$ and $U_{2} \in \mathcal{R}$ such that

$$
\operatorname{cl}_{\mathcal{R}}\left(U_{2}\left(x_{2}\right)\right) \subset A_{2} \cap U_{1}\left(x_{1}\right) \cap S_{2}\left(x_{2}\right) .
$$


Hence, by induction, it is clear that there exist sequences $\left(x_{n}\right)_{n=1}^{\infty}$ and $\left(U_{n}\right)_{n=1}^{\infty}$ in $X$ and $\mathcal{R}$, respectively, such that

$$
\operatorname{cl}_{\mathcal{R}}\left(U_{n}\left(x_{n}\right)\right) \subset A_{n} \cap U_{n-1}\left(x_{n-1}\right) \cap S_{n}\left(x_{n}\right)
$$

for all $n \in \mathbb{N}$.

Hence, since by the reflexivity of $\mathcal{R}$ we have

$$
x_{n} \in U_{n}\left(x_{n}\right) \subset \operatorname{cl}_{\mathcal{R}}\left(U_{n}\left(x_{n}\right)\right)
$$

for all $n \in \mathbb{N}$, it is clear that $\left(U_{n}\left(x_{n}\right)\right)_{n=1}^{\infty}$ is a decreasing, infinitesimal sequence of nonvoid subsets of $X(\mathcal{S})$. Therefore, by Theorem 6.10 , we have

$$
\bigcap_{n=1}^{\infty} \operatorname{cl}_{\mathcal{R}}\left(U_{n}\left(x_{n}\right)\right) \neq \emptyset \text {. }
$$

Moreover, by the corresponding inclusions and the definition of $A$, it is clear that we also have

$$
\bigcap_{n=1}^{\infty} \operatorname{cl}_{\mathcal{R}}\left(U_{n}\left(x_{n}\right)\right) \subset \bigcap_{n=1}^{\infty} A_{n} \cap U_{n-1}\left(x_{n-1}\right) \subset \bigcap_{n=1}^{\infty} A_{n} \cap U_{0}\left(x_{0}\right)=A \cap U_{0}\left(x_{0}\right) .
$$

Therefore, the required assertion $A \cap U_{0}\left(x_{0}\right) \neq \emptyset$ is also true.

From Theorem 9.5, by Theorems 7.3 and 7.10, it is clear that in particular we also have the following

Theorem 8.5. If $\mathcal{R}$ is a topological, topologically filtered, topologically regular relator on $X$ such that $\mathcal{R}$ is either topologically relatively locally sequentially compact, or uniformly countable and properly sequentially convergence-adherence complete, then $\mathcal{R}$ is a Baire relator.

Remark 8.6. Note that the sets $U_{n}\left(x_{n}\right)$ used in the proof of Theorem 9.4 are, in addition, open. Therefore, by introducing some weaker completeness and compactness properties of relators, Theorems 9.4 and 9.5 could be sharpened.

First of all, the pseudocompactness properties of relators should be be investigated. Namely, according to Engelking [6, p.264], a Tychonoff space $X$ is pseudocompact if and only if $\bigcap_{n=1}^{\infty} \operatorname{cl}\left(A_{n}\right) \neq \emptyset$ for any decreasing sequence $\left(A_{n}\right)_{n=1}^{\infty}$ of nonvoid open subsets of $X$.

\section{References}

[1] J. M. Aarts and D. J. Lutzer, Completeness properties designed for recognizing Baire spaces, Dissertationes Math. 1161974 1-43.

[2] N. Bourbaki, General Topology, Chapters 5-10 Springer-Verlag, Berlin, 1989.

[3] E. Čech, On bicompact spaces, Annals Math. 38 1937, 823-844. 
[4] Á. Császár, General Topology, Akadémiai Kiadó, Budapest, 1978.

[5] A. S. Davis, Indexed systems of neighborhoods for general topological spaces, Amer. Math. Monthly 68, 1961, 886-893.

[6] R. Engelking, General Topology, Polish Scientific Publisher, Warszawa, 1977.

[7] Z. Frolík, Baire spaces and some generalizations of complete metric spaces, Czech. Math. J. 11, 1961, 237-247.

[8] R. C. Haworth and R. A. McCoy, Baire spaces, Dissertationes Math. 141, 1977, $1-73$.

[9] J. Mala, Relators generating the same generalized topology, Acta Math. Hungar. 60, 1992, 291-297.

[10] J. Mala and Á. Száz, Properly topologically conjugated relators, Pure Math. Appl. 3 1992, 119-136.

[11] J. Mala and Á. Száz, Modifications of relators, Acta Math. Hungar. 77 1997, 69-81.

[12] H. Nakano and K. Nakano, Connector theory, Pacific J. Math. 56 1975, 195-213.

[13] G. Pataki, Supplementary notes to the theory of simple relators, Radovi Mat. 9 1999, 101-118.

[14] G. Pataki, On the extensions, refinements and modifications of relators, Math. Balkanica 15 2001, 155-186.

[15] G. Pataki and Á. Száz, A unified treatment of well-chainedness and connectedness properties, Acta Math. Acad. Paedagog. Nyházi. (N.S.) 19 2003, 101-165.

[16] W. J. Pervin, Quasi-uniformization of topological spaces, Math. Ann. 1471962 , 316-317.

[17] Cs. Rakaczki and Á. Száz Semicontinuity and closedness properties of relations in relator spaces, Mathematica (Cluj) 452003.

[18] Á. Száz, Coherences instead of convergences, Proc. Conf., Convergence and Generalized Functions (Katowice, 1983), Inst. Math. Polish Acad. Sci. (Warsaw, 1984), 141-148.

[19] Á. Száz, Basic tools and mild continuities in relator spaces, Acta Math. Hungar. 50 1987, 177-201.

[20] Á. Száz, Directed, topological and transitive relators, Publ. Math. Debrecen 35 1988, 179-196.

[21] Á. Száz, Lebesgue relators, Monathsh. Math. 110 1990, 315-319.

[22] Á. Száz, Relators, Nets and Integrals, Unfinished doctoral thesis, Debrecen, 1991, $1-126$.

[23] Á. Száz, Structures derivable from relators, Singularité 3 1992, 14-30. 
[24] Á. Száz, Inverse and symmetric relators, Acta Math. Hungar. 60 1992, 157-176.

[25] Á. Száz, Refinements of relators, Tech. Rep., Inst. Math. Inf., Univ. Debrecen 1993/76, 1-19.

[26] Á. Száz, Cauchy nets and completeness in relator spaces, Colloq. Math. Soc. János Bolyai 55 1993, 479-489.

[27] Á. Száz, Neighbourhood relators, Bolyai Soc. Math. Stud. 4 1995, 449-465.

[28] Á. Száz, Topological characterizations of relational properties, Grazer Math. Ber. 327 1996, 37-52.

[29] Á. Száz, Uniformly, proximally and topologically compact relators, Math. Pannon. 8 1997, 103-116.

[30] Á. Száz, An extension of Kelley's closed relation theorem to relator spaces, Filomat (Nis) 14 2000, 49-71.

[31] Á. Száz, Somewhat continuity in a unified framework for continuities of relations, Tatra Mt. Math. Publ. 24 2002, 41-56.

[32] Á. Száz, Upper and lower bounds in relator spaces, Serdica Math. J. 292003 , 2391-270.

[33] Á. Száz, Rare and meager sets in relator spaces, Tatra Mt. Math. Publ.

Institute of Mathematics, University of Debrecen, H-4010 Debrecen, Pfi2,

Hungary szazmath.klte.hu 